\title{
Biological effects and clinical characteristics of microRNA-106a in human colorectal cancer
}

\author{
YUZHENG HE $^{1,2}$, GUIQI WANG ${ }^{1}$, LEI ZHANG ${ }^{1}$, \\ CONGJIE ZHAI ${ }^{1}$, JUN ZHANG ${ }^{1}$, XUSHENG ZHAO ${ }^{1}$, XIA JIANG ${ }^{1}$ and ZENGREN ZHAO ${ }^{1}$ \\ ${ }^{1}$ Department of General Surgery, The First Hospital of Hebei Medical University; \\ ${ }^{2}$ Department of Thoracic Surgery, The Second Hospital of Hebei Medical University, \\ Shijiazhuang, Hebei 050031, P.R. China
}

Received September 24, 2015; Accepted March 14, 2017

DOI: $10.3892 / \mathrm{ol} .2017 .6179$

\begin{abstract}
MicroRNAs serve important roles in various diseases, particularly cancer. microRNA-106a (miR-106a) exhibits abnormal expression and oncogenic activity in carcinogenesis. The clinical significance of the abnormal expression of miR-106a in colorectal cancer is poorly understood. In the present study, miR-106a expression from colorectal cancer tissues was quantified using the reverse transcription-quantitative polymerase chain reaction. The overexpression or knockdown of miR-106a was performed by transfection with microRNA mimic or inhibitor in human colorectal carcinoma HCT116 cells. The overexpression of miR-106a promoted viability and inhibited apoptosis in colorectal cancer cells. The association between miR-106a expression and clinicopathological factors was analyzed, and it was identified that miR-106a exhibited significantly increased expression in adenocarcinoma tissues compared with in mucinous carcinoma tissues, and the expression of miR-106a was identified to be associated with the depth of invasion and differentiation. The expression of miR-106a in plasma was also determined and it was identified that increased expression of miR-106a, as a characteristic of patients with colorectal cancer, may be distinguished from that of other patients by digitization of the areas under the receiver operating characteristic curves. These data suggested that miR-106a is a potential biomarker in the diagnosis of colorectal carcinoma. However, the underlying
\end{abstract}

Correspondence to: Professor Zengren Zhao, Department of General Surgery, The First Hospital of Hebei Medical University, 89 Donggang Road, Shijiazhuang, Hebei 050031, P.R. China E-mail: zzr-doctor@163.com

Abbreviations: miRNA, microRNA; miR-106a, miRNA-106a; CRC, colorectal cancer; CCK-8, Cell Counting kit-8; PI, propidium iodide; FITC, fluorescein isothiocyanate

Key words: microRNA-106a, colorectal cancer, microRNA, cell apoptosis, cell viability, clinicopathological factors molecular mechanism of miR-106a-promoted viability and inhibition of apoptosis requires further investigation.

\section{Introduction}

Colorectal cancer (CRC) is one of the three most common types of cancer worldwide and is a major contributor to cancer-associated mortality (1). Effective screening to detect cancer is expected to decrease the mortality rate of CRC (2). Novel methods are currently under development for the detection of CRC, including those based on the detection of microRNAs (miRNA/miR) $(3,4)$.

miRNAs are small (between 19 and 22 nucleotides) non-coding RNA molecules that are encoded by eukaryotic genomic DNA. miRNAs lead to translation repression or degradation of specific target mRNAs by directly binding to their potential target site in the $3^{\prime}$ untranslated region (5). Located in the spacer regions between protein-coding genes or in the introns of protein-encoding genes, miRNA coding sequences are transcribed as primary miRNAs in the same manner as the mRNAs of the protein-coding genes (6). miRNAs are important mediators of biological functions, and their dysregulation has been implicated in a wide range of diseases, including malignancies, heart diseases, inflammation and lung diseases (7-9).

As identified previously, miR-28 suppresses viability, but activates metastasis in CRC cells (10), miR-381 increases radiosensitivity in esophageal squamous cell carcinoma (11), and miRNA-27b targets vascular endothelial growth factor C to inhibit tumor progression and angiogenesis in CRC (12).

miR-106a (miRBase accession number MIMAT0000103), located in Xq26.2, exhibits oncogenic activity in humans, and its expression is often altered in carcinogenesis (13). It has been demonstrated to be highly expressed in cancer tissues, including gastric carcinoma and ovarian cancer, and may be a potential biomarker in the diagnosis of certain malignant tumors (14-16).

In the present study, the expression levels of miR-106a were determined in CRC tissues and plasma, compared with the control group, and the potential association between the expression of miR-106a and clinicopathological factors of all patients was analyzed. In order to investigate the underlying molecular mechanism for the effects of miR-106, apoptosis of 
CRC cells transfected with miR-106a mimic and miR-106a inhibitor was examined using flow cytometry.

\section{Patients and methods}

Patients. In total, 42 patients with CRC (23 male, 19 female; median age, 63 years) and 42 healthy individuals (24 male, 18 female; median age, 60 years) were recruited from The First Hospital of Hebei Medical University (Shijiazhuang, China) between October 2013 and March 2014. No previous local or systemic treatment had been administered to the 42 patients with CRC prior to surgery. Written informed consent was obtained from all of the patients, in accordance with the protocols of the Ethics Review Board at Hebei Medical University (Shijiazhuang, China), which approved the present study.

Samples. A total of 42 pairs of CRC and non-tumor adjacent tissues were obtained from patients that underwent radical resection between October 2013 and March 2014 at The First Hospital of Hebei Medical University. The corresponding normal tissues were obtained from a section of the resected specimen $\geq 5 \mathrm{~cm}$ from the edge of the tumor. The samples were stored in liquid nitrogen or an $-80^{\circ} \mathrm{C}$ freezer until use.

Plasma samples were collected from $42 \mathrm{CRC}$ patients prior to surgical resection and 7 days following surgery respectively. Furthermore, 42 plasma samples were obtained from 42 cases of age-matched healthy individuals as the control. Cell-free plasma was extracted from all blood samples within $2 \mathrm{~h}$ of collection using a two-step protocol at 2,140 $\mathrm{x}$ g for $10 \mathrm{~min}$ and $12,840 \times \mathrm{g}$ for $2 \mathrm{~min}$. Plasma was transferred to fresh tubes and stored in $\mathrm{a}-80^{\circ} \mathrm{C}$ freezer until use.

Cell culture. The human CRC cell line HCT116 was obtained from Professor Xiaofeng Sun (Division of Oncology, Department of Clinical and Experimental Medicine, Faculty of Health Sciences, Linköping University, Linköping, Sweden). Cells were cultured in McCoy's 5A medium (Gibco; Thermo Fisher Scientific, Inc., Waltham, MA, USA) containing $10 \%$ fetal bovine serum (Gibco; Thermo Fisher Scientific, Inc.) and $1 \%$ penicillin-streptomcyin (Invitrogen; Thermo Fisher Scientific, Inc.) in a $37^{\circ} \mathrm{C}$ humidified incubator containing $5 \% \mathrm{CO}_{2}$.

Quantitative miRNA analysis. Total RNA was extracted from the frozen tissues using TRIzol reagent (Invitrogen; Thermo Fisher Scientific, Inc.), according to the manufacturer's protocol, and resuspended in $60 \mu \mathrm{l}$ pre-heated $\left(95^{\circ} \mathrm{C}\right)$ nuclease-free water.

Total RNA was extracted from $400 \mu 1$ plasma samples using the miRNeasy Serum/Plasma kit (Qiagen $\mathrm{GmbH}$, Hilden, Germany), according to the manufacturer's protocol, and eluted with $105 \mu \mathrm{l}$ pre-heated $\left(95^{\circ} \mathrm{C}\right)$ double distilled water.

The concentration and purity of all RNA samples were detected using a NanoDrop ND-2000 spectrophotometer (NanoDrop Technologies; Thermo Fisher Scientific, Inc.). All RNA was stored at $-80^{\circ} \mathrm{C}$ until use following determination of the concentration RNA using a spectrophotometer.

Reverse transcription was carried out using the One Step PrimeScript ${ }^{\circledR}$ miRNA cDNA Synthesis kit (Takara Bio, Inc., Otsu, Japan), according to the manufacturer's protocol.
Quantitative polymerase chain reaction (qPCR) was performed using an All-in-One ${ }^{\mathrm{TM}}$ miRNA qPCR kit (QP101; GeneCopoeia, Inc., Guangzhou, China) with an ABI 7500 Real-Time PCR system (Applied Biosystems; Thermo Fisher Scientific, Inc.), according to the manufacturer's protocol. All-in-OneTM miRNA qPCR Primers were used, including a hsa-miR-106a-5p primer pair (HmiRQP0026) and a HsnRNA U6 primer pair (HmiRQP9001; GeneCopoeia, Inc.). The PCR reaction mixture included $10 \mu \mathrm{l} 2 \mathrm{X}$ All-in-One ${ }^{\mathrm{TM}}$ qPCR mix, $2 \mu$ All-in-One ${ }^{\mathrm{TM}}$ miRNA qPCR Primer, $2 \mu 1$ Universal Adaptor PCR Primer, $2 \mu \mathrm{l}$ template cDNA and $4 \mu \mathrm{l}$ double distilled water, for a total volume of $20 \mu \mathrm{l}$. The reaction mixture was incubated at $95^{\circ} \mathrm{C}$ for $10 \mathrm{~min}$, followed by 40 cycles of $95^{\circ} \mathrm{C}$ for $15 \mathrm{sec}$ and $60^{\circ} \mathrm{C}$ for $60 \mathrm{sec}$. All RT-qPCR reactions were performed in triplicate.

U6 small nuclear RNA was used as a housekeeping gene to normalize miRNA expression, and the relative expression of miR-106a in CRC cells was determined using the comparative threshold cycle $(\mathrm{Cq})$ method $\left(2^{-\Delta \Delta \mathrm{Cq}}\right)(17)$.

Cell Counting kit-8(CCK-8) assays. HCT116 cells were seeded into 96-well plates and then transfected with miR-106a mimic (miR10000103-1-5), miR-106a mimic control (miR01101-1-5), miR-106a inhibitor (miR20000103-1-5) or miR-106a inhibitor control (miR02101-1-5; all RiboBio, Guangzhou, China) using Lipofectamine 2000 (Invitrogen; Thermo Fisher Scientific, Inc.). At $24 \mathrm{~h}$ after culture, the cells were harvested and absorbance values at 450 were determined using CCK-8 reagent (Dojindo Molecular Technologies, Inc., Kumamoto, Japan) according to the manufacturer's protocol. Tests were performed in triplicate.

Apoptosis assay. Fluorescein isothiocyanate (FITC)-conjugated Annexin $\mathrm{V}$ and propidium iodide (PI) staining were performed by means of the Annexin V-FITC Apoptosis Detection kit (Neobioscience, Shenzhen, China). Following transfection with miR-106a mimic or inhibitor, or negative control, cells were cultured for $48 \mathrm{~h}$, resuspended in $1 \mathrm{X}$ binding buffer, washed twice with PBS, and treated with Annexin V-FITC and PI. Following $10 \mathrm{~min}$ of incubation at room temperature in the dark, cells were submitted to flow cytometry with a BD FACSCalibur flow cytometer calibrated with CaliBRITE beads, and the results were analyzed using CellQuest software (all BD Biosciences, San Jose, CA, USA). Tests were performed in quadruplicate.

Early apoptotic cells were located in the lower-right quadrant, late apoptotic or necrotic cells were located in the upper-right quadrant, normal cells were located in the lower-left quadrant and mechanically damaged cells were located in the upper-left quadrant of the flow cytometric dot plots (18).

Statistical analysis. Each experiment was conducted at least three times. All values are presented as the median and 25th and 75 th percentiles or the mean \pm standard deviation. Differences were tested for significance using a Student's t-test, unpaired t-test, Mann-Whitney U test or a two-way analysis of variance followed by Tukey's post hoc test in SPSS software (version 19.0; IBM SPSS, Armonk, NY, USA). P<0.05 was considered to indicate a statistically significant difference. 


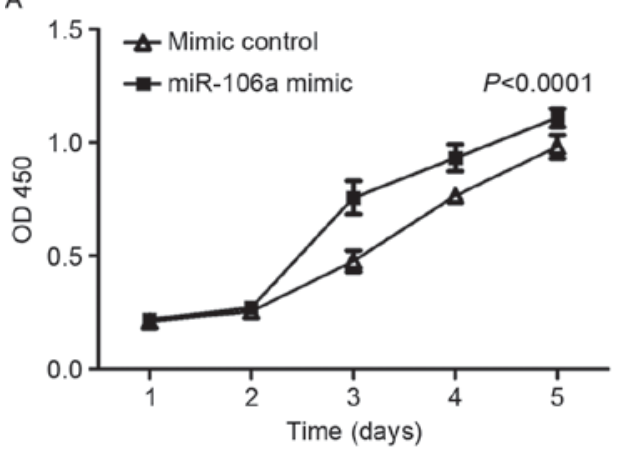

C

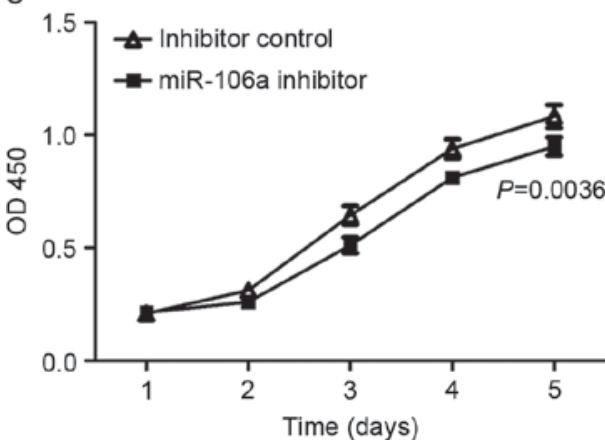

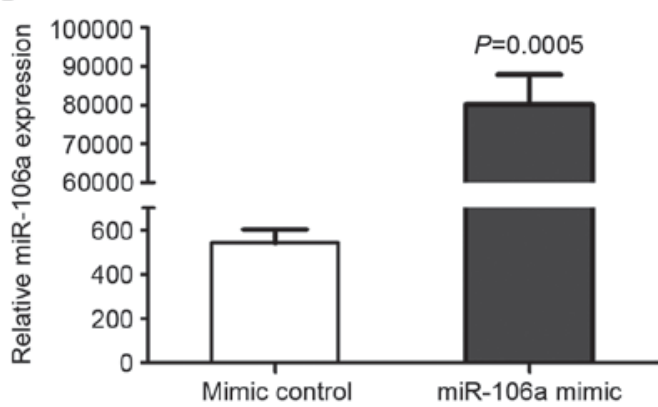

D

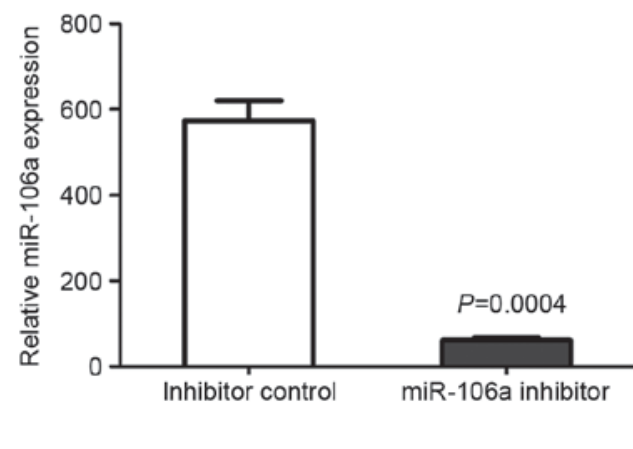

Figure 1. Effects of miR-106a on the viability of colorectal cancer cells. (A) Cell viability of HCT116 cells transfected with miR-106a mimic or mimic control. (B) Relative expression of miR-106a in HCT116 cells transfected with miR-106a mimic or mimic control. (C) Cell viability of HCT116 cells transfected with miR-106a inhibitor or inhibitor control. (D) Relative expression of miR-106a in HCT116 cells transfected with miR-106a inhibitor or inhibitor control. P-values are expressed in comparison with the respective controls (Student's t-test). miR-106a, microRNA-106a; OD, optical density.

\section{Results}

Effects of miR-106a on CRC cell viability. Our previous study (19) identified that miR-106a expression was increased in the plasma of patients with CRC compared with healthy controls. Therefore, it was hypothesized that miR-106a expression may be associated with the progress of CRC. In the present study, the effects of miR-106a on cell viability and apoptosis in CRC cells were analyzed. First, to measure the effect of miR-106a on cell viability, a CCK-8 assay was carried out. HCT116 cells were transfected with miR-106a mimic or mimic control, miR-106a inhibitor or inhibitor control. Cell viability curves and, at $24 \mathrm{~h}$ after transfection, miR-106a expression were examined (Fig. 1).

The expression of miR-106a and cell viability were significantly upregulated by the miR-106a mimic comparison with mimic control in HCT116 cells $(\mathrm{P}<0.01$; Fig. $1 \mathrm{~A}$ and $\mathrm{B}$, respectively). Conversely, following transfection with miR-106a inhibitor, the viability of HCT116 cells was also significantly decreased compared with inhibitor control-transfected cells $(\mathrm{P}=0.0036$; Fig. 1C) and the expression of miR-106a was significantly decreased $(\mathrm{P}=0.0004$; Fig. 1D). These results indicated that the miR-106a expression level was positively associated with CRC cell viability.

Effects of miR-106a on CRC cell apoptosis. The function of miR-106a on cell apoptosis was determined using flow cytometry. HCT116 cells were transfected with miR-106a mimic or mimic control, miR-106a inhibitor or inhibitor control. At $48 \mathrm{~h}$ after transfection, the apoptosis rates of HCT116 cells were determined (Fig. 2).
Following transfection with miR-106a mimic or mimic control, cell status was determined using flow cytometry, with early apoptotic cells located in the lower right quadrant of the plot (Fig. 2A and B). HCT116 cells transfected with miR-106a mimic exhibited a lower rate of early apoptosis compared with the control group. The mean \pm standard deviation early apoptosis rates were significantly decreased for the miR-106a mimic compared with the mimic control $(8.68 \pm 0.75$ vs. $12.45 \pm 1.16 \%$, respectively; $\mathrm{P}=0.0016$; $\mathrm{n}=4$; Fig. $2 \mathrm{C}$ ).

Flow cytometry was also employed to analyze cells subsequent to transfection with an miR-106a inhibitor or an inhibitor control (Fig. 2D and E). HCT116 cells transfected with miR-106a inhibitor exhibited a higher rate of early apoptosis compared with the control group. The mean \pm standard deviation early apoptosis rates were significantly increased for the miR-106a inhibitor compared with the inhibitor control $(8.803 \pm 0.27$ vs. $6.733 \pm 0.60 \%$, respectively; $\mathrm{P}=0.0162 ; \mathrm{n}=4$; Fig. 2F).

These results indicated that the miR-106a mimic decreased early apoptosis in HCT116 cells, and that the miR-106a inhibitor increased early apoptosis in HCT116 cells, suggesting that miR-106a promoted cell viability by inhibiting early apoptosis in CRC cells.

Expression of miR-106a is upregulated in human CRC tissues and plasma. In previous studies (19), miR-106a expression was upregulated in the serum of patients with CRC. In the present study, the expression of miR-106a in human CRC tissues and plasma was determined. It was identified that the expression level of miR-106a was significantly upregulated in tumor tissues compared with adjacent tissues 
Table I. Association between miR-106a expression in cancer tissues and clinicopathological characteristics of patients with colorectal cancer.

\begin{tabular}{|c|c|c|c|}
\hline Variables & Patients $(n=42)$ & $\begin{array}{l}\text { miR-106a expression, median } \\
\text { (25th and } 75 \text { th percentile) }\end{array}$ & P-value \\
\hline Age, years & & & 0.5800 \\
\hline$\leq 63$ & 21 & $26.643(13.741,72.249)$ & \\
\hline$>63$ & 21 & $25.934(14.554,40.273)$ & \\
\hline Sex & & & 0.6133 \\
\hline Male & 23 & $26.643(16.049,50.062)$ & \\
\hline Female & 19 & $25.934(12.790,47.944)$ & \\
\hline Dukes & & & 0.6311 \\
\hline $\mathrm{A}, \mathrm{B}$ & 19 & $26.643(16.049,74.260)$ & \\
\hline $\mathrm{C}, \mathrm{D}$ & 23 & $25.934(13.316,47.944)$ & \\
\hline Pathological type & & & $0.0423^{\mathrm{a}}$ \\
\hline Adenocarcinoma & 36 & $30.493(15.941,49.824)$ & \\
\hline Mucinous carcinoma & 6 & $11.939(4.928,32.244)$ & \\
\hline Depth of invasion & & & $0.0431^{\mathrm{a}}$ \\
\hline $\mathrm{T} 1, \mathrm{~T} 2$ & 9 & $37.528(27.910,286.856)$ & \\
\hline $\mathrm{T} 3, \mathrm{~T} 4$ & 33 & $24.340(13.223,48.528)$ & \\
\hline Location & & & 0.2610 \\
\hline Colon & 21 & $18.367(12.665,42.628)$ & \\
\hline Rectum & 21 & $39.325(22.801,80.544)$ & \\
\hline Lymph node metastasis & & & 0.4375 \\
\hline Absent & 23 & $26.643(15.791,70.237)$ & \\
\hline Present & 19 & $25.934(13.316,46.261)$ & \\
\hline Distant metastasis & & & 0.8700 \\
\hline Absent & 33 & $26.643(14.129,50.144)$ & \\
\hline Present & 9 & $23.090(14.460,48.162)$ & \\
\hline Differentiation & & & $0.0382^{\mathrm{a}}$ \\
\hline Poor & 8 & $12.539(5.423,33.613)$ & \\
\hline Moderate-well & 34 & $35.731(20.439,60.149)$ & \\
\hline
\end{tabular}

${ }^{a} \mathrm{P}<0.05$, Mann-Whitney U test. miR-106a, microRNA-106a.

[median (25th percentile, 75th percentile, $26.2882(14.5358$, 49.3492) vs. 8.7187 (5.4891, 13.6726); P<0.0001; Fig. 3A]. The association between miR-106a expression in cancer tissues and clinicopathological characteristics of patients with CRC was also analyzed (Table I).

miR-106a expression in plasma of patients with CRC or healthy controls, and pre- or post-surgery in patients with $\mathrm{CRC}$, was also investigated. The expression level of miR-106a in plasma was significantly higher in patients with CRC compared with in the healthy control group, consistent with our previous study (19) $(\mathrm{P}=0.0041$; Fig. 3B). The healthy control group contained 42 age-matched healthy individuals with patients with CRC (data not shown). miR-106a expression in plasma post-surgery was decreased compared with in plasma pre-surgery, but no statistically significant difference was identified $(0.16 \pm 0.18$ vs. $8.80 \pm 40.50 ; \mathrm{P}=0.1600$; Fig. $3 \mathrm{C})$. No significant association was identified between miR-106a expression in plasma and the clinicopathological parameters of the patients with CRC (data not shown). The sensitivity and specificity of miR-106a were analyzed using a receiver operating characteristic curve in CRC diagnosis (Fig. 3D).

miR-106a expression in CRC tissues is associated with differentiation and pathological type. The expression of miR-106a in CRC tissues was identified to be associated with the differentiation and pathological type of tumor, by analyzing the clinicopathological characteristics of CRC patients. The expression level of miR-106a in CRC tissues was significantly increased in patients with adenocarcinoma compared with in patients with mucinous carcinoma $(100.96 \pm 262.20$ vs. $18.14 \pm 18.22$; $\mathrm{P}=0.0348$; Fig. $4 \mathrm{~A}$ ). miR-106a expression in CRC tissues was also increased in patients exhibiting moderate-well differentiation compared with that in patients exhibiting poor differentiation (108.87 \pm 272.79 vs. $18.12 \pm 19.20$; $\mathrm{P}=0.0343$; Fig. 4B).

The underlying molecular mechanism of increased miR-106a expression in adenocarcinoma and moderate-well differentiation $\mathrm{CRC}$ requires further investigation. No significant association 
A

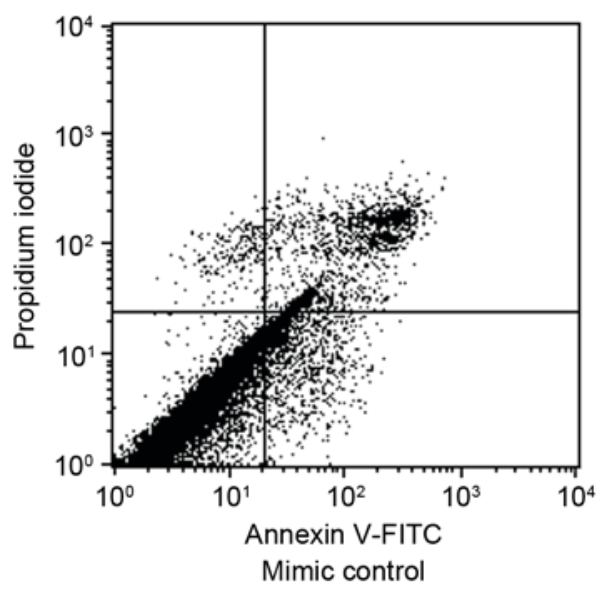

C

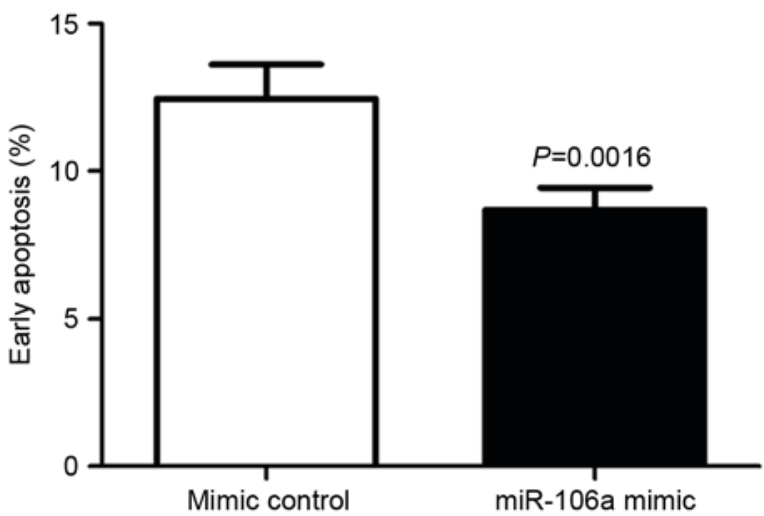

B
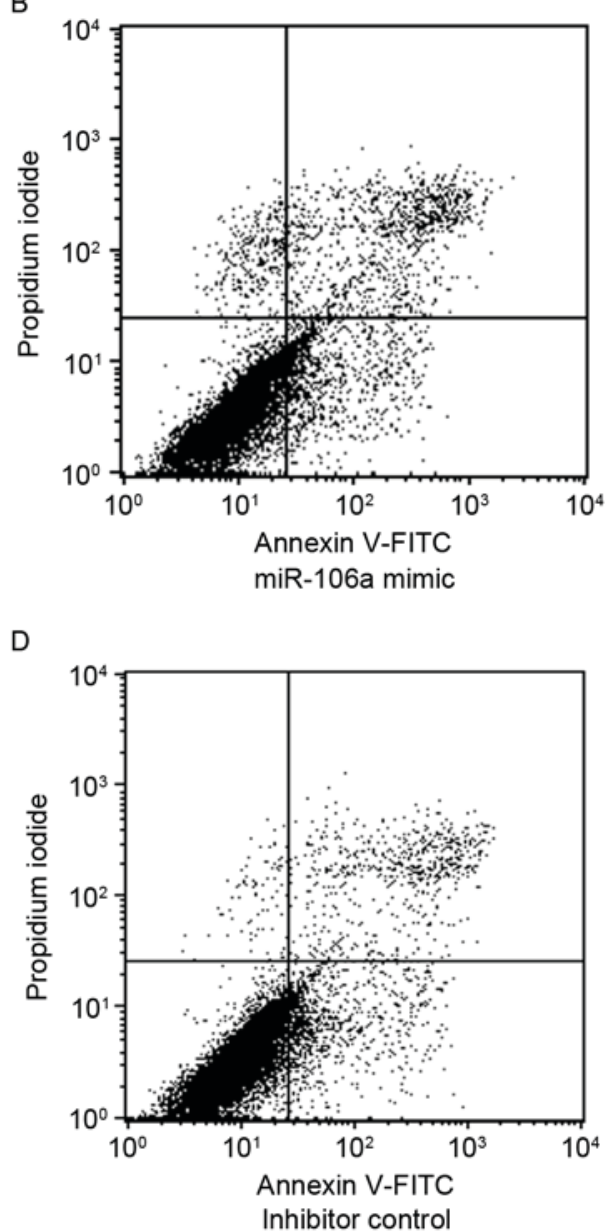

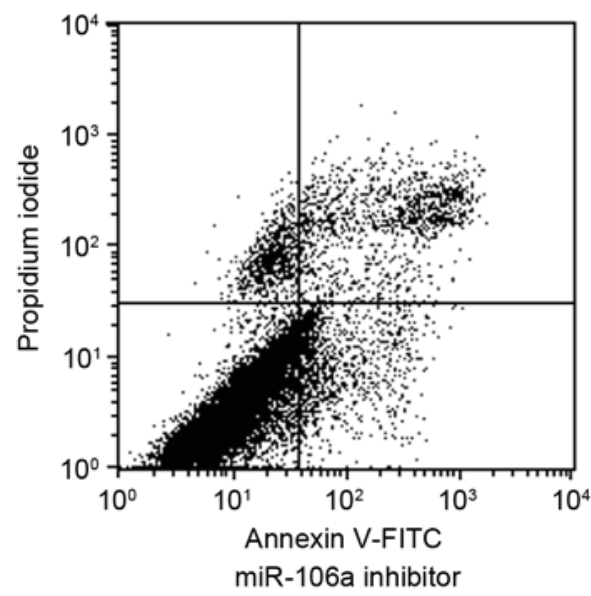

$\mathrm{F}$

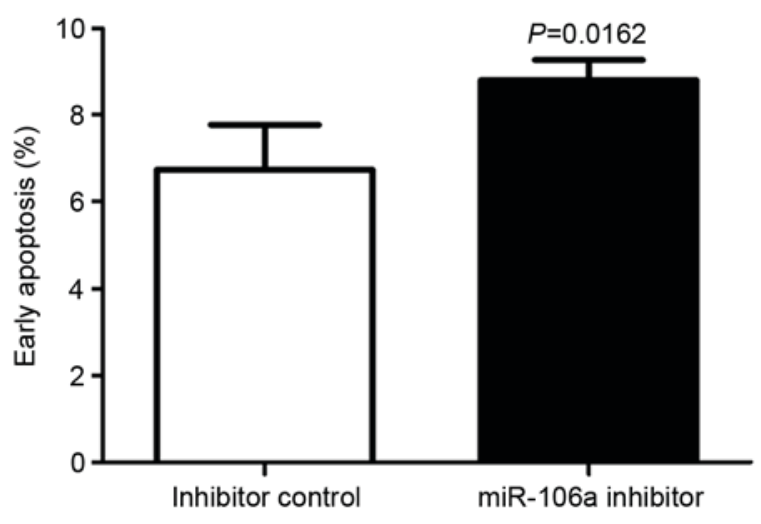

Figure 2. Effects of miR-106a on apoptosis of colorectal cancer cells. HCT116 cells were transfected with (A) mimic control or (B) miR-106a mimic, and (C) the proportion of cells in early apoptosis was quantified. HCT116 cells were transfected with (D) inhibitor control or (E) miR-106a inhibitor mimic, and (F) the proportion of cells in early apoptosis was quantified. Results are representative of at least 3 independent experiments. P-values were calculated with an unpaired t-test and are expressed in comparison with the respective controls. miR-106a, microRNA-106a; FITC, fluorescein isothiocyanate.

was identified between miR-106a expression and other clinicopathological characteristics, such as sex, age, distant metastasis, Dukes staging, tumor location and lymph node metastasis.

\section{Discussion}

miR-106a was identified to serve an oncogenic role in carcinogenesis $(20,21)$. miR-106a enhanced the invasiveness of human glioma stem cells, pancreatic cancer, gastric cancer and glioblastoma (22-24).

The results of the present study indicated that overexpression of miR-106a by transfection with miR-106a mimic increased the viability and suppressed early apoptosis of HCT116 cells. Knocking down the miR-106a expression by miR-106a inhibitor identified that cell viability was inhibited and the number of pro-apoptotic cells was upregulated. These 
A

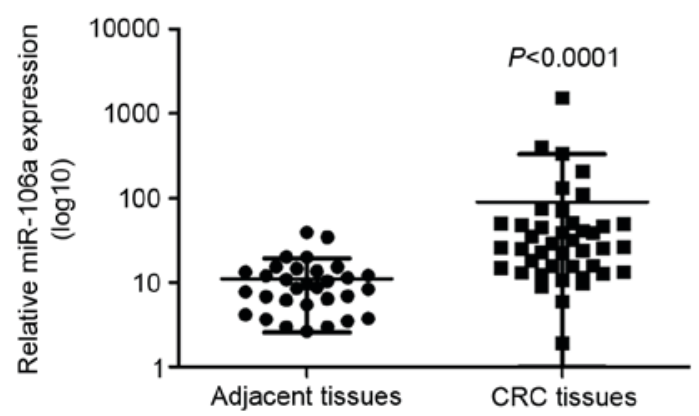

C

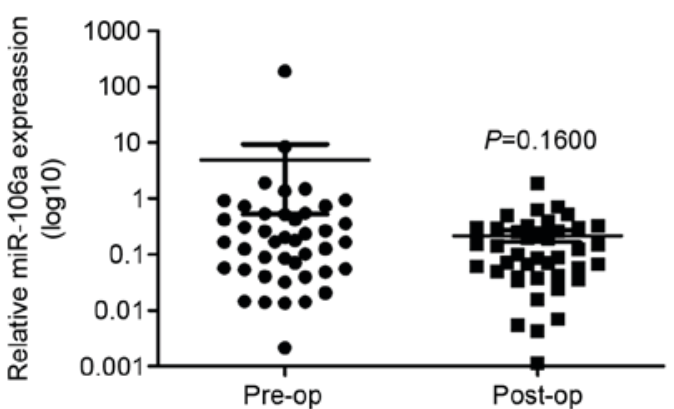

B

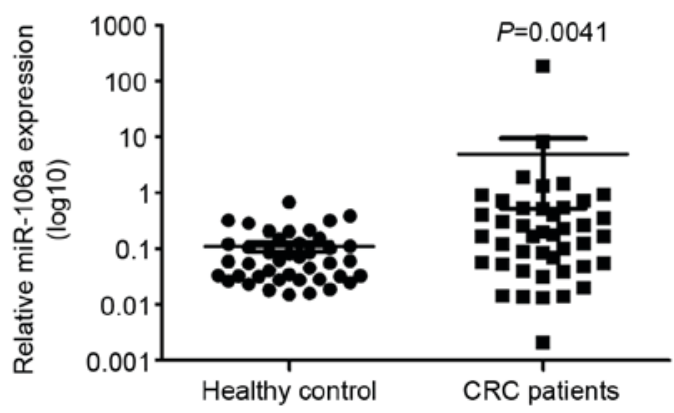

D

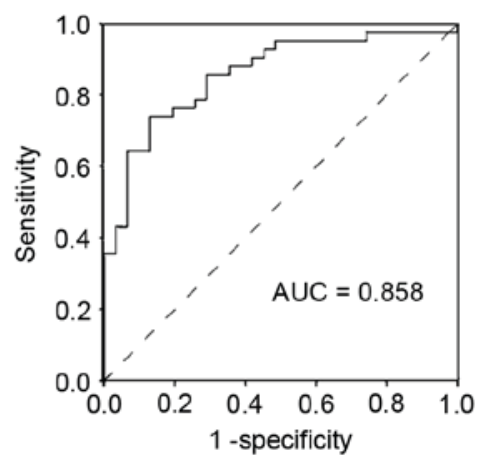

Figure 3. Expression of miR-106a is upregulated in human CRC tissues and plasma. miR-106a expression was determined in (A) CRC tissues and adjacent tissues, (B) plasma of patients with CRC and healthy controls, and (C) plasma of pre-surgery patients and post-surgery patients. (D) Receiver operating characteristic curve for the miR-106a discriminating patients with CRC from healthy controls. P-values are expressed in comparison with the respective controls (Mann-Whitney U test). miR-106a, microRNA-106a; CRC, colorectal cancer; op, operation; AUC, area under the curve.

A

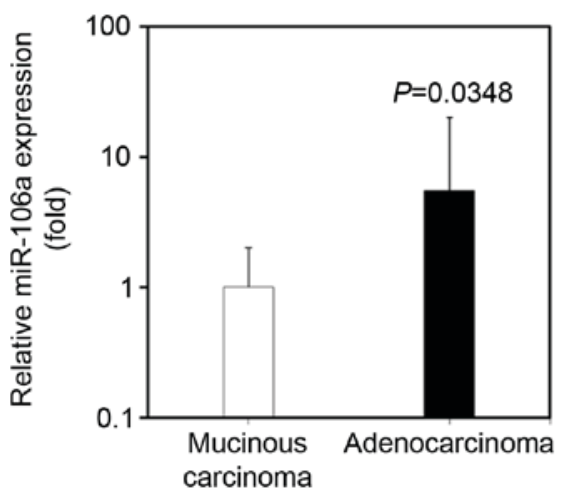

B

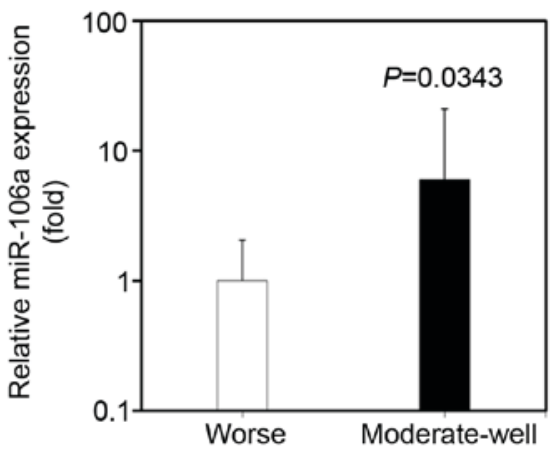

Figure 4. Expression of miR-106a is associated with differentiation and pathological type in CRC tissues. (A) miR-106a expression in colorectal adenocarcinoma and colorectal mucinous carcinoma tissues. (B) miR-106a expression in poorly differentiated CRC tissues and moderately-well differentiated CRC tissues. P-values are expressed in comparison with the respective controls (Student's t-test).

results suggested that miR-106a served an important role in the viability and apoptosis of CRC cells.

miR-106a expression was determined in CRC tissues, and the association between miR-106a expression and clinicopathological data was analyzed. The expression level of miR-106a in CRC tissues was significantly upregulated in CRC tissues compared with adjacent tissues. More importantly, an increased association was identified between miR-106a expression and pathological type, differentiation and depth of invasion. The expression levels of miR-106a were increased in adenocarcinoma compared with mucinous carcinoma $(\mathrm{P}=0.0423)$. The expression of miR-106a was identified to be positively associated with differentiation. The well-differentiated CRC tissues exhibited an increased expression level of miR-106a compared with poorly differentiated CRC tissues $(\mathrm{P}=0.0382)$. However, miR-106a expression was not identified to be associated with other clinicopathological characteristics, including sex, age, distant metastasis, Dukes staging, tumor location and lymph node metastasis $(\mathrm{P}>0.05)$. These results are in contrast with a previous study identifying that miR-106a exhibited an increased expression level in rectal tumors (25).

A number of studies have focused on circulating miRNAs, which have been reported as an effective and non-invasive biomarker for detecting various cancers or other diseases (26-28). 
In our previous study, the plasma level of miR-106a was significantly increased in patients with CRC compared with healthy controls (19). The expression of miR-106a was also determined in the plasma of 40 patients with CRC and 40 healthy individuals, and the expression level of miR-106a was significantly increased in patients with CRC compared with in the healthy control group. In the present study, although the plasma levels of miR-106a were analyzed in patients with CRC at different clinical status (age, tumor size, tumor-node-metastasis stage or metastasis), no significant difference was identified. No significant alterations in the miR-106a expression were identified following surgery compared with its preoperative level, which is in contrast with previous results (19). It is hypothesized that this may be due to the limited sample size. Therefore, expanding the sample size in future research may provide more insight into the underlying molecular mechanism.

It was hypothesized that miR-106a served an important role in carcinogenesis of CRC. In the present study, the focus was on analyzing the effect of miR-106a in CRC cell apoptosis, viability and the alterations in miR-106a expression in CRC tissues.

miR-106a may be a potential biomarker in the diagnosis of CRC, particularly in the diagnosis of colorectal adenocarcinoma. miR-106a serves important roles in the differentiation of colorectal adenocarcinoma, CRC progression, CRC cell viability and apoptosis. miR-106a may be associated with the migration and invasion of cancer cells. Increased accuracy could be obtained if a number of types of circulating miRNAs are combined to provide a clinical diagnosis of CRC. Further research is required to elucidate the role of miR-106a in tumor progression and its complicated interactions with oncogenes and tumor suppressors. Further study is required to elucidate the underlying molecular mechanism of miR-106a in the development of CRC.

\section{Acknowledgements}

The authors are indebted to Professor Xiaofeng Sun (Linköping University, Linköping, Sweden) for generously providing the HCT116 cells. The present study was supported by the National Natural Science Foundation of China (grant no. 81072034), the Excellent Youth Foundation of Hebei Scientific Committee (grant no. 2013206252), the International Science and Technology Cooperation Program of China (grant no. 2014DFA31150) and Hebei Province (grant no. 12396105D).

\section{References}

1. Wasserman M, Baxter NN, Rosen B, Burnstein M and Halverson AL: Systematic review of internet patient information on colorectal cancer surgery. Dis Colon Rectum 57: 64-69, 2014.

2. Yang W, Lee DY and Ben-David Y: The roles of microRNAs in tumorigenesis and angiogenesis. Int $\mathbf{J}$ Physiol Pathophysiol Pharmacol 3: 140-155, 2011.

3. Hamaya Y, Yoshida K, Takai T, Ikuma M, Hishida A and Kanalka S: Factors that contribute to faecal cyclooxygenase-2 mRNA expression in subjects with colorectal cancer. Br J Cancer 102: 916-921, 2010

4. Mo ZH, Wu XD, Li S, Fei BY and Zhang B: Expression and clinical significance of microRNA-376a in colorectal cancer. Asian Pac J Cancer Prev 15: 9523-9527, 2014.

5. Fayyad-Kazan H, Bitar N, Najar M, Lewalle P, Fawad-Kazan M, Badran R, Hamade E, Daher A, Hussein N, ElDirani R, et al: Circulating miR-150 and miR-342 in plasma are novel potential biomarkers for acute myeloid leukemia. J Transl Med 11: 31, 2013
6. Ye JJ and Cao J: MicroRNAs in colorectal cancer as markers and targets-Recent advances. World J Gastroenterol 20: 4288-4299, 2014.

7. Oglesby IK, McElvaney NG and Greene CM: MicroRNAs in inflammatory lung disease-master regulators or target practice? Respir Res 11: 148, 2010.

8. Holloway JW, Savarimuthu Francis S, Fong KM and Yang IA: Genomics and the respiratory effects of air pollution exposure. Respirology 17: 590-600, 2012

9. Small EM and Olson EN: Pervasive roles of microRNAs in cardiovascular biology. Nature 469: 336-342, 2011.

10. Almeida MI, Nicoloso MS, Zeng L, Ivan C, Spizzo R, Gafa R, Xiao L, Zhang X, Vannini I, Fanini F, et al: Strand-specific miR-28-5p and miR-28-3p have distinct effects in colorectal cancer cells. Gastroenterology 142: 886-896, 2012.

11. Zhou S, Ye W, Ren J, Shao Q, Qi Y, Liang J and Zhang M: MicroRNA-381 increases radiosensitivity in esophageal squamous cell carcinoma. Am J Cancer Res 5: 267-277, 2014.

12. Ye J, Wu X, Wu D, Wu P, Ni C, Zhang Z, Chen Z, Qiu F, Xu J and Huang J: miRNA-27b targets vascular endothelial growth factor $\mathrm{C}$ to inhibit tumor progression and angiogenesis in colorectal cancer. PLoS One 8: e60687, 2013.

13. Tong AW and Nemunaitis J: Modulation of miRNA activity in human cancer: A new paradigm for cancer gene therapy? Cancer Gene Ther 15: 341-355, 2008 .

14. Xiao B, Guo J, Miao Y, Jiang Z, Huan R, Zhang Y, Li D and Zhong J: Detection of miR-106a in gastric carcinoma and its clinical significance. Clin Chim Acta 400: 97-102, 2009.

15. Xie X, Liu HT, Mei J, Ding FB, Xiao HB, Hu FQ, Hu R and Wang MS: miR-106a promotes growth and metastasis of non-small cell lung cancer by targeting PTEN. Int J Clin Exp Pathol 8: 3827-3834, 2015.

16. Kim YW, Kim EY, Jeon D, Liu JL, Kim HS, Choi JW and Ahn WS: Differential microRNA expression signatures and cell type-specific association with Taxol resistance in ovarian cancer cells. Drug Des Devel Ther 8: 293-314, 2014.

17. Livak KJ and Schmittgen TD: Analysis of relative gene expression data using real-time quantitative PCR and the 2(-Delta Delta C(T)) method. Methods 25: 402-408, 2001.

18. Yu D, Zhang S, Du W, Zhang J, Fan Z, Hao H, Liu Y, Zhao X, Qin T and Zhu H: Expression of intracellular interferon-alpha confers antiviral properties in transfected bovine fetal fibroblasts and does not affect the full development of SCNT embryos. PLoS One 9: e94444, 2014.

19. Zhang L, Meng L, Fan Z, Liu B, Pei Y and Zhao Z: Expression of plasma miR-106a in colorectal cancer and its clinical significance. Nan Fang Yi Ke Da Xue Xue Bao 34: 354-357, 2014 (In Chinese).

20. Wang Z, Liu M, Zhu H, Zhang W, He S, Hu C, Quan L, Bai J and $\mathrm{Xu}$ N: miR-106a is frequently upregulated in gastric cancer and inhibits the extrinsic apoptotic pathway by targeting FAS. Mol Carcinog 52: 634-646, 2013.

21. Yuan R, Zhi Q, Zhao H, Han Y, Gao L, Wang B, Kou Z, Guo Z, $\mathrm{He} \mathrm{S}$, Xue $\mathrm{X}$ and $\mathrm{Hu} \mathrm{H}$ : Upregulated expression of miR-106a by DNA hypomethylation plays an oncogenic role in hepatocellular carcinoma. Tumour Biol 36: 3093-3100, 2015.

22. Li P, Xu Q, Zhang D, Li X, Han L, Lei J, Duan W, Ma Q, Wu Z and Wang Z: Upregulated miR-106a plays an oncogenic role in pancreatic cancer. FEBS Lett 588: 705-712, 2014.

23. Wang Z, Wang B, Shi Y, Xu C, Xiao HL, Ma LN, Xu SL, Yang L, Wang QL, Dang WQ, et al: Oncogenic miR-20a and miR-106a enhance the invasiveness of human glioma stem cells by directly targeting TIMP-2. Oncogene 34: 1407-1419, 2015.

24. Zhu M,Zhang N and He S: Similarly up-regulated microRNA-106a in matched formalin-fixed paraffin-embedded and fresh frozen samples and the dynamic changes during gastric carcinogenesis and development. Pathol Res Pract 210: 909-915, 2014.

25. Schee K, Boye K, Abrahamsen TW, Fodstad $\varnothing$ and Flatmark K: Clinical relevance of microRNA miR-21, miR-31, miR-92a, miR-101, miR-106a and miR-145 in colorectal cancer. BMC Cancer 12: 505, 2012.

26. Criscitiello C, Sotiriou C and Ignatiadis M: Circulating tumor cells and emerging blood biomarkers in breast cancer. Curr Opin Oncol 22: 552-558, 2010.

27. Cheng H, Zhang L, Cogdell DE, Zheng H, Schetter AJ, Nykter M, Harris CC, Chen K, Hamilton SR and Zhang W: Circulating plasma MiR-141 is a novel biomarker for metastatic colon cancer and predicts poor prognosis. PLoS One 6: e17745, 2011.

28. Qin X, Xu H, Gong W and Deng W: The tumor cytosol miRNAs, fluid miRNAs, and exosome miRNAs in lung cancer. Front Oncol 4: 357, 2015. 